\title{
Avaliação da qualidade de vida e do consumo alimentar de funcionários de uma empresa de fornecimento de energia elétrica
}

\section{Quality Assessment of Life and food intake of employees of a company supply electricity}

\author{
Helena Marina Sebastião \\ Universidade Tecnológica Federal do Paraná - UTFPR - Ponta Grossa - PR - Brasil \\ helena_sbas@yahoo.com.br \\ Marcelo Santiago Timossi \\ Universidade Tecnológica Federal do Paraná - UTFPR - Ponta Grossa - PR - Brasil \\ marcelo_timossi@hotmail.com \\ Eliana Aparecida Fagundes Queiroz Bortolozo \\ Universidade Tecnológica Federal do Paraná - UTFPR - Ponta Grossa - PR - Brasil \\ bortolozopg@gmail.com \\ Luiz Alberto Pilatti \\ Universidade Tecnológica Federal do Paraná - UTFPR - Ponta Grossa - PR - Brasil \\ lapilatti@utfpr.edu.br
}

\section{RESUMO}

OBJETIVO: Avaliar a relação dos hábitos alimentares com a qualidade de vida de trabalhadores de uma empresa de fornecimento de energia.

MÉTODOS: Para desenvolvimento da pesquisa foram avaliados os itens qualidade de vida (através do WHOQOL-Bref) e consumo alimentar (por meio do Questionário de Frequência Alimentar QFA), em 50 trabalhadores de numa empresa de fornecimento de energia, localizada na cidade de Ponta Grossa, no estado do Paraná - Brasil.

RESULTADOS: A população envolvida na pesquisa, na sua maioria, relatou possuir uma boa qualidade de vida, porém os resultados obtidos indicaram prevalência de dieta que necessita de modificações. Grupos alimentares como frutas e leguminosas são ingeridos com pouca frequência, enquanto que grupos de bebidas açucaradas e gorduras são consumidos além do preconizado.

CONCLUSÕES: Não sendo observada relação entre percepção de qualidade de vida e hábito alimentar, torna-se importante a necessidade de se incluírem nos programas de promoção da qualidade de vida dos trabalhadores temas relacionados a hábitos alimentares saudáveis.

PALAVRAS-CHAVE: Qualidade de vida. Consumo alimentar. Saúde do trabalhador. Doenças crônicas não transmissíveis. 


\section{ABSTRACT}

OBJECTIVE: Evaluate the relationship of dietary habits with the quality of life of workers in a company providing energy.

METHODS: To develop the research evaluated the items quality of life (through the WHOQOLBref) and food intake (through the Food Frequency Questionnaire - FFQ), in 50 workers from a power supply company, located in the city of Ponta Grossa in the state of Paraná - Brazil.

RESULTS: The results showed a population that mostly reported owning a good quality of life, but with a diet that requires changes in some aspects, to prevent the development of chronic diseases and balancing quality of life.

CONCLUSIONS: No observed relationship between perception of quality of life and eating habits, it is important the need to include in programs to promote quality of life of workers topics related to healthy eating habits.

KEYWORDS: Quality of life. Food consumption. Occupational health. Chronic noncommunicable diseases.

\section{Introdução}

Partindo-se da premissa de que os adultos ativos passam maior parte do tempo da sua vida no ambiente de trabalho, é evidente a necessidade de se buscar uma adequada qualidade de vida (QV), não apenas no âmbito da habitualidade, mas também no ambiente de trabalho. Vale ressaltar que saúde não significa somente a ausência de doenças, mas abrange o bem-estar do indivíduo como um todo, envolvendo tanto a sua capacidade de adaptação ao meio quanto a sua estabilidade física e mental (NESPECA; CYRILLO, 2011; BJÖRKLUND; JENSEN; LOHELA-KARLSSON, 2013).

Indivíduos que possuem em sua rotina alimentação saudável, hábitos de vida ativa e boas relações sociais, podem alcançar uma adequada QV em geral, bem como no seu ambiente de trabalho, beneficiando, no caso, a sua produtividade (FRANCO; CASTRO; WOLKOFF, 2013).

O que, entretanto, observa-se na atualidade é que o homem vem se tornando cada vez mais sedentário, em função das tecnologias que transmudaram as atividades que detinham maior dispêndio de energia. $\mathrm{O}$ fato tem influência sobre o nível de atividade física, aumentando os riscos de desenvolvimento de diversas doenças (DU et al., 2013). Além dessa inatividade, o desequilíbrio emocional do indivíduo, em função da intensa competição das empresas em busca de lucros e destaque, tem igualmente repercutido no seu bem-estar (BJÖRKLUND; JENSEN; LOHELAKARLSSON, 2013).

As mudanças quanto às escolhas alimentares dos trabalhadores acabam afetadas pelo seu estilo de vida, levando-os a adotarem hábitos alimentares inadequados, tais como a ingestão de dietas com alta densidade energética, que contribui diretamente para elevada incidência, entre esta população, de doenças crônicas não transmissíveis (DCNT), como por exemplo: obesidade, doenças cardíacas e dislipidemias (DUNCAN et al., 2012; BEZERRA et al., 2013; SALINARDI et al., 2013).

Se a hipótese de que indivíduos que fazem escolhas alimentares mais adequadas apresentam melhor QV, uma vez que estas influenciam positivamento no seu estado de saúde, for correta, torna-se inegável a correlação da QV com as condutas alimentares de trabalhadores. Com base nesta realidade, este estudo teve como objetivo avaliar a relação dos hábitos alimentares com a qualidade de vida (QV) de trabalhadores de uma empresa de fornecimento de energia. 


\section{Metodologia}

Este estudo, de caráter quali-quantitativo, foi realizado numa empresa de fornecimento de energia, localizada na cidade de Ponta Grossa, no estado do Paraná - Brasil. A amostra foi composta por 50 profissionais, de ambos os sexos, do setor de eletroeletrônica e de eletromecânica.

Respeitando-se os critérios éticos pertinentes, a pesquisa foi submetida e aprovada pelo Comitê de Ética em Pesquisa envolvendo Seres Humanos da Universidade Tecnológica Federal do Paraná (CEP/UTFPR), registrada com Certificado de Apresentação para Apreciação Ética (CAAE) n. 21637113.3.0000.5547.

Para mensuração da qualidade de vida foi utilizado o instrumento WHOQOL-Bref (THE WHOQOL GROUP, 1996). Os resultados foram analisados através de uma ferramenta desenvolvida por Pedroso et al. (2010).

Para avaliação do comportamento alimentar dos trabalhadores, foi aplicado um Questionário de Frequência Alimentar (QFA) adaptado do intrumento proposto por Fisberg e Marchioni (2012). Utilizou-se a pirâmide alimentar proposta por Philippi et al. (1999), ajustada para o Brasil, como fonte de base para a distribuição dos diferentes alimentos contidos no QFA Os alimentos foram alocados em dez grupos alimentares (cereais, leite e derivados, hortaliças, frutas, bebidas açucaradas, carnes, gorduras, leguminosas, guloseimas, bebidas alcoólicas), dependendo das suas respectivas características nutricionais. Na sequência, foi feita efetuada uma análise relacionando o grupo alimentar com a frequência (\%) do seu consumo.

\section{Resultados}

A amostra, que correspondeu a aproximadamente $10,00 \%$ do quadro de funcionários que compõem a regional da cidade em pauta, foi composta por adultos, na sua grande maioria do sexo masculino $(92,00 \%)$, com faixa etária entre 30 e 55 anos de idade.

Pelo fato da unidade estudada não contar com refeitório, os funcionários recebem Vale alimentação no valor de R \$ 25,00 por refeição, para um almoço e um jantar. Assim sendo, 100,00\% dos indivíduos avaliados não possuíam um local fixo para a realização de suas refeições durante o turno do trabalho.

O Gráfico 1 apresenta os resultados referentes a QV em geral, bem como em seus domínios, numa escala de 0 a 100.

Gráfico 1 - Avaliação da QV geral e seus domínios, em uma amostra de trabalhadores de uma empresa de fornecimento de energia

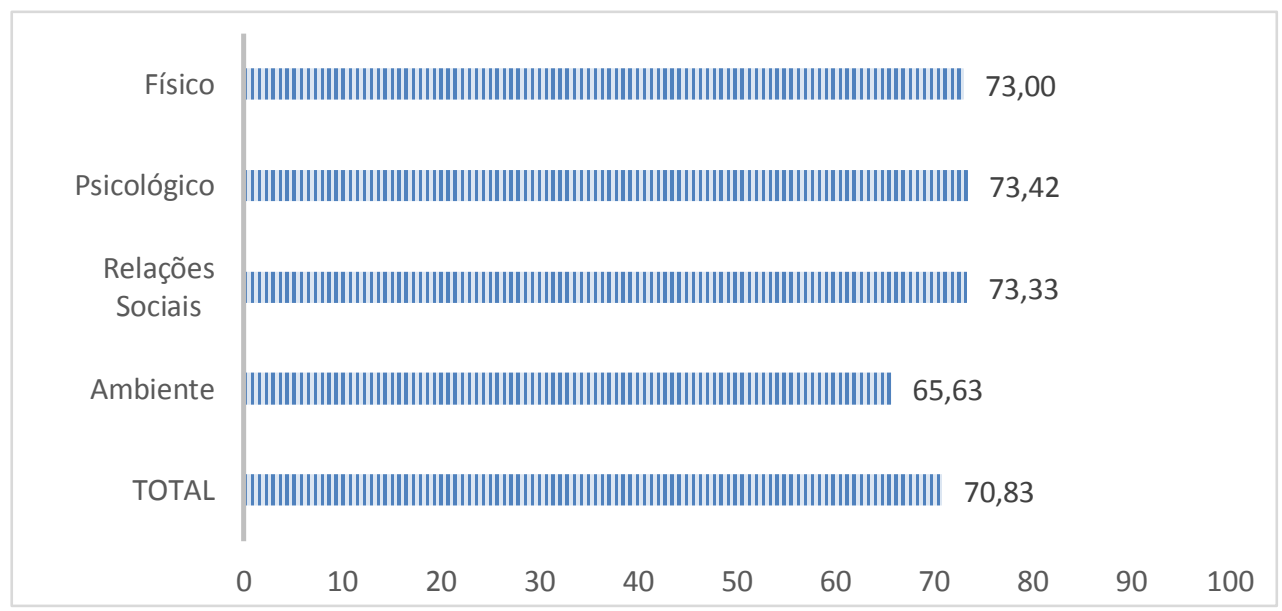

Fonte: Autoria própria (2013). 
O Gráfico 2 apresenta os valores resultantes da análise da frequência de consumo diário dos grupos alimentares. A maior frequência $(25,62 \%)$ foi observada no grupo dos cereais (cereais e derivados, raízes e tubérculos), seguindo-se o grupo das bebidas $(18,05 \%)$, leite e derivados $(11,65 \%)$, hortaliças $(11,36 \%)$, gorduras $(7,72 \%)$, carnes $(6,70 \%)$, guloseimas $(6,40 \%)$, frutas $(6,11 \%)$, leguminosas $(5,67 \%)$ e, por último, o grupo das bebidas alcoólicas $(0,72 \%)$.

Gráfico 2 - Análise da frequência de consumo diário dos grupos alimentares em uma amostra de trabalhadores de uma empresa de fornecimento de energia

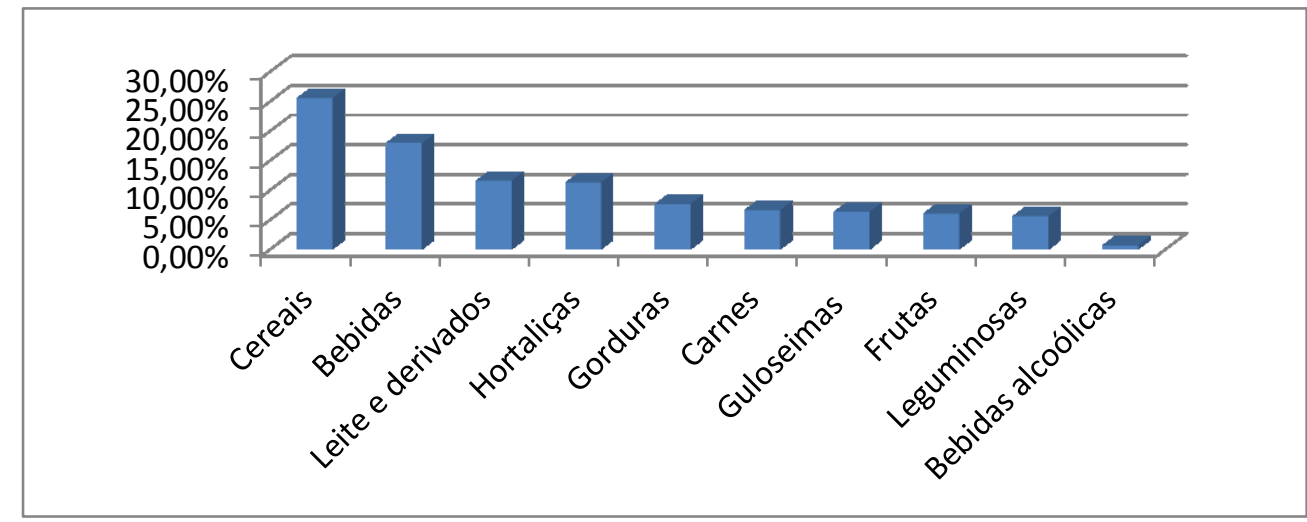

Fonte: Autoria própria (2013).

\section{Discussão}

De modo geral, foi constatado índice satisfatório para a qualidade de vida global, com com pontuação superior a 70,00. Os domínios psicicológico, físico e relações sociais atingiram pontuações similares, na casa de 73 . O domínio ambiente $(65,63)$ apresentou a menor pontuação, sendo o único com escore inferior a 70,00. Costa et al. (2012), ao avaliarem QV de trabalhadores de uma indústria, utilizando o instrumento WHOQOL-Bref, também identificaram menor pontuação no domínio ambiente. Leão et al. (2011), ao estudarem trabalhadores de uma empresa fornecedora de energia, também observaram a mesma tendência.

A partir da análise da frequência de consumo diário dos grupos alimentares, verificou-se na amostra estudada uma prevalência de padrão alimentar necessitando de modificações. Contribuíram para este resultado: a baixa ingestão dos grupos alimentares representados pelas leguminosas e frutas, bem como a maior frequência no consumo de bebidas adoçadas com açúcar.

O baixo consumo de leguminosas demonstra que o feijão, alimento tradicional da cultura brasileira, tem sido consumido com baixa frequência. As leguminosas constituem fontes de proteínas, minerais, vitaminas e fibras e oferecem vário benefícios à saúde (VENN; MANN, 2004; BRASIL, 2006). Inquéritos alimentares entre a população brasileira demonstraram tendências a baixo consumo de leguminosas e outros vegetais, a par de maior consumo de açucarados e gorduras (LEVY-COSTA et al., 2005; BRASIL, 2006; MONTEIRO et al., 2011; LEVY-COSTA et al., 2012).

O grupo de bebidas açucaradas, como os refrigerantes e sucos industrializado, foi relatado como segundo grupo alimentar mais consumido. Mas, a ingestão excessiva destes alimentos é apontada como importante fator de risco para doenças metabólicas (QI et al., 2012; AMBROSINI et al., 2013).

A ingestão de guloseimas, cujo consumo é mais frequente que o de leguminosas e de frutas, pode estar relacionada com a obesidade e outras DCNTs (BRASIL, 2006; WORLD HEALTH ORGANIZATION, 2003). Segundo estudos que analisaram dados da Pesquisa de Orçamento Familiar (POF) 2002/03, a contribuição do açúcar adicionado aos alimentos dobrou, graças especialmente ao maior consumo de refrigerantes e biscoitos. A POF 2008-2009 comprovou que o limite máximo de açúcares livres foi largamente ultrapassado em todas as classes de 
rendimentos, tanto no meio urbano, quanto no rural $(16,10 \%$ e $17,10 \%$, respectivamente) (LEVYCOSTA et al., 2005; LEVY-COSTA et al., 2012).

O consumo do grupo de gorduras, representado por alimentos gordurosos, constituiu outro ponto negativo, uma vez que foi o quinto grupo alimentar mais consumido. O Guia Alimentar intitulado Pirâmide Alimentar preconiza consumo apenas esporádico deste grupo de alimentos (PHILIPPI et al., 1999). Assim como os alimentos açucarados, o excessivo consumo de alimentos gordurosos, observado no presente estudo, pode representar fator de risco para o desenvolvimentos das DCNTs (LEVY-COSTA et al., 2012, BRASIL, 2006).

No que tange aos resultados positivos encontrados na avaliação do padrão alimentar dos trabalhadores estudados, destaca-se o fato de que os grupos fontes de carboidrato complexo (pães, cereais, raízes e tubérculos); fontes de proteína (leite e derivados) e de vitaminas, minerais e fibras (hortaliças) estão situados entre os quatro itens mais frequentemente consumidos. Recomenda-se que o grupo de pães, cereais, raízes e tubérculos, alocados na base da Pirâmide Alimentar, seja a principal fonte energética da dieta (PHILIPPI et al., 1999). Há, porém uma tendência entre a população brasileira de redução deste grupo de alimentos e aumento do consumo de outras fontes energéticas, com base de alimentos açucarados e gordurosos (BRASIL, 2006; LEVY-COSTA et al., 2012). No caso do grupo das hortaliças, que é fonte de vitaminas e de minerais, o fato de ter ficado entre os mais frequentemente consumidos é satisfatório, visto que comumente é observada baixa ingestão destes alimentos pela população brasileira (IBGE, 2004).

O grupo de leites e derivados e das carnes, que constituem fontes de proteínas, vitaminas e minerais, responsável pela construção, desenvolvimento e manutenção do organismo, apresentou uma frequência adequada de consumo. Entretanto, não foi investigado neste estudo se tais alimentos eram mais frequentemente ingeridos sob a forma magra ou com redução de gordura. Alimentos de origem animal podem também ser fontes de gordura saturada, a qual é muitas vezes responsável pela ocorrência das DCNTs (BRASIL, 2006; WORLD HEALTH ORGANIZATION, 2003). Em estudo realizado por Levy-Costa et al. (2012), o excesso de gordura saturada em domicílios urbanos foi consistente com a maior participação de produtos de origem animal (carne bovina, frango, embutidos, leite e derivados). Torna-se, portanto, necessário cuidados constantes sobre o consumo de alimentos pertencentes a estes grupos alimentares, buscando-se sempre preferência pelas opções mais saudáveis, em vista de se assegurar a saúde.

O baixo consumo de bebidas alcoólicas observado neste estudo destoa de registros brasileiros que demonstram aumento significativo do consumo de bebidas com teor alcoólico (LEVY-COSTA et al., 2005; LEVY-COSTA et al., 2012).

Ao serem avaliados em conjunto os resultados obtidos na avaliação da QV e do padrão alimentar, nota-se que não houve relação entre ambos, o que sugere que os trabalhadores podem não estar associando uma dieta saudável com QV. Apesar da maioria dos indivíduos relatarem boa QV, o padrão alimentar geral não foi satisfatório. Este dado corrobora a pesquisa realizada por Nespeca e Cyrillo (2011), que apontou ter uma parcela da amostra em estudo (45,70\%) relatado uma situação de boa QV associada com uma dieta necessitando de alteração.

\section{Considerações finais}

Este estudo efetuou uma avaliação da percepção de trabalhadores de uma empresa de fornecimento de energia, no que se refere à respectiva $\mathrm{QV}$, relacionando este dado com os hábitos alimentares.

De acordo com os dados obtidos na pesquisa, a maioria dos trabalhadores avaliados têm percepção positiva da sua QV. Entretanto, ao ser avaliado o padrão alimentar dos participantes, foram observadas algumas inadequações na dieta. Grupos alimentares, como frutas e leguminosas são ingeridos com pouca frequência, enquanto grupos de bebidas açucaradas e gorduras são ingeridos com frequências maiores que o preconizado. Assim, a inserção de programas voltados à QV do trabalhador, devem contemplar temas relacionados à promoção da alimentação saudável, uma vez que não foi observada a relação entre percepção da QV com padrão alimentar. 
Em face do que foi apurado, investigações mais detalhadas fazem-se necessárias, no intuito de se obterem resultados mais fidedignos em relação aos valores quantitativos da ingestão de alimentos, assim como permitir comparações com parâmetros de recomendação nutricional. $\mathrm{O}$ presente estudo analisou a frequência de consumo (número de vezes consumido), de acordo com determinada periodicidade, podendo ter subestimado/superestimado certos grupos de alimentos, já que não avaliou a quantidade de ingestão, nem porções consumidas.

\section{Referências}

AMBROSINI, G. L.; ODDY, W. H.; HUANG, R. C.; MORI, T. A.; BEILIN, L. J. JEBB, S. A. Prospective associations between sugar-sweetened beverage intakes and cardiometabolic risk factors in adolescents. The American Journal of Clinical Nutrition, v. 98, n. 2, p. 327-334, May 2013. crossef

BEZERRA, I. N.; SOUZA, A. M.; PEREIRA, R. A.; SICHIERI, R. Consumo de alimentos fora do domicílio no Brasil. Revista de Saúde Pública, v. 47, supl. 1, p. 200s-211s, fev. 2013. Disponível em: $<$ http://www.scielo.br/scielo.php?pid $=$ S0034-89102013000700006\&script $=$ sci_arttext $>$. Acesso em: 22 dez. 2014.

BJÖRKLUND, C.; JENSEN, I.; LOHELA-KARLSSON, M. Is a change in work motivation related to a change in mental well-being?. Journal of Vocational Behavior, v. 83, n. 3, p. 571-580, Dec. 2013. crossef

BRASIL. Ministério da Saúde. Guia alimentar para a população brasileira: promovendo a alimentação saudável. Brasília: Ministério da Saúde, 2006.

COSTA, C. S. N.; FREITAS, E. G.; SOUZA MENDONÇA, L. C.; ALEM, M. E. R.; COURY, H. J. C. G. Capacidade para o trabalho e qualidade de vida de trabalhadores industriais. Ciência e Saúde Coletiva, v. 17, n. 6, p. 1635-1642, 2012. Crossef

DU, H.; LI, L.; WHITLOCK, G.; BENNETT, D.; GUO, Y.; BIAN, Z.; CHEN, J.; SHERLIKER, P.; HUANG, Y.; ZHANG, N.; ZHENG, X.; LI, Z.; HU, R.; COLLINS, R.; PETO, R.; CHEN, Z.; China Kadoorie Biobank Collaborative Group. Physical activity and sedentary leisure time and their associations with BMI, waist circumference, and percentage body fat in 0.5 million adults: the China Kadoorie Biobank study. The American Journal of Clinical Nutrition, v. 97, n. 3, p. 487496, Mar. 2013. rossef

DUNCAN, B. B.; CHOR, D.; AQUINO, E. M. L.; BENSENOR, I. M.; MILL, J. G.; SCHMIDT, M. I; LOTUFO, P. A.; VIGO, A.; BARRETO, S. M. Doenças crônicas não transmissíveis no Brasil: prioridade para enfrentamento e investigação. Revista de Saúde Pública, São Paulo, v. 46,

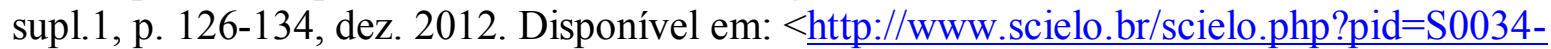
$89102012000700017 \&$ script $=$ sci arttext $>$. Acesso em: 17 ago. 2013.

FISBERG, R. M.; MARCHIONI, D. M. L. Manual de avaliação do consumo alimentar em estudos populacionais: a experiência do inquérito de saúde em São Paulo (ISA). Grupo de Pesquisa de Avaliação de Consumo Alimentar (GAC), Faculdade de Saúde Pública. São Paulo: Universidade de São Paulo, 2012.

FRANCO, A. S.; CASTRO, I. R. R.; WOLKOFF, D. B. Impacto da promoção sobre consumo de frutas e hortaliças em ambiente de trabalho. Revista de Saúde Pública, São Paulo, v. 47, n. 1, p. 29-36, fev. 2013. Disponível em: $<$ http://www.scielo.br/scielo.php?pid=S003489102013000100005\&script=sci arttext $>$. Acesso em: 22 dez. 2014. 
IBGE. INSTITUTO BRASILEIRO DE GEOGRAFIA E ESTATÍSTICA. Coordenação de Índices de Preços. Pesquisa de Orçamentos Familiares 2002-2003: análise da disponibilidade domiciliar e estado nutricional no Brasil. Rio de Janeiro: IBGE, 2004.

LEÃO, H. F. P.; GOMES, S. A.; ALMEIDA, A. H. S.; CASTRO, P. J. P.; TASHIRO, T.; BATISTA, G. R. Qualidade de vida e nível de atividade física de trabalhadores com diferentes tempos de serviço. Revista Brasileira de Ciências da Saúde, v. 15, n. 1, p. 31-38, 2011. Crosšef

LEVY-COSTA, R. B.; SICHIERI, R.; PONTES, N. dos S.; MONTEIRO, C. A. Disponibilidade domiciliar de alimentos no Brasil: distribuição e evolução (1974-2003). Revista de Saúde Pública, São Paulo, v. 39, n. 4, p. 530-540, ago. 2005. Disponível em:

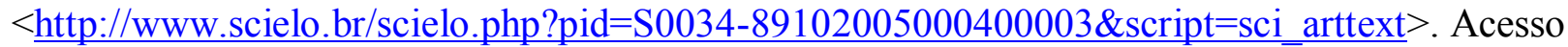
em: 17 dez. 2014.

LEVY-COSTA, R. B.; CLARO, R. M.; MONDINI, L.; SICHIERI, R.; MONTEIRO, C. A. Distribuição regional e socioeconômica da disponibilidade domiciliar de alimentos no Brasil em 2008-2009. Revista de Saúde Pública, São Paulo, v. 46, n. 1, p. 6-15, fev. 2012. Disponível em: $<$ http://www.rsp.fsp.usp.br/mensagem/ pub/edicao_atual. tpl.php>. Acesso em: 11 nov. 2013.

MONTEIRO, C. A.; LEVY, R. B.; CLARO, R. M.; CASTRO, I. R. R. DE; CANNON, G. Increasing consumption of ultra-processed foods and likely impact on human health: evidence from Brazil. Public Health Nutrition, v. 14, n. 1, p. 5-13, Jan. 2011. Disponível em:

$<$ http://www.wphna.org/htdocs/downloadsdec2012/2011_PHN_Monteiro_et_al.pdf $>$. Acesso em: 22 dez. 2014.

NESPECA, M.; CYRILLO, D. C. Qualidade de vida no trabalho de funcionários públicos: papel da nutrição e da qualidade de vida. Acta Scientiarum Health Sciences, v. 33, n. 2, p. 187-195, jul./dez. 2011. crossef

PEDROSO, B.; PILATTI, L. A.; GUTIERREZ, G. L.; PICININ, C. T. Cálculo dos escores e estatística descritiva do WHOQOL-Bref através do Microsoft Excel. Revista Brasileira de Qualidade de Vida, Ponta Grossa, v. 2, n. 1, p. 31-36, jan/jun. 2010. Disponível em: $<$ http://revistas.utfpr.edu.br/pg/index. php/rbqv/ article/view/687/505>. Acesso em: 22 dez. 2014.

PHILIPPI, S. T.; LATTERZA, A. R.; CRUZ, A. T. R.; RIBEIRO, L. C. Pirâmide alimentar adaptada: guia para escolha dos alimentos. Revista de Nutrição, v. 12, n. 1, p. 65-80, jan./abr. 1999. Disponível em: $<$ http://www.scielo.br/scielo.php?script=sci arttext\&pid=S141552731999000100006>. Acesso em: 22 dez. 2014.

QI, Q.; CHU, A. Y.; KANG, J. H.; JENSEN, M. K.; CURHAN, G. C.; PASQUALE, L. R.; RIDKER, P. M.; HUNTER, D. J.; WILLETT, W. C.; RIMM, E. B.; CHASMAN, D. L.; HU, F. B.; QI, L. Sugar-sweetened beverages and genetic risk of obesity. The New England Journal of Medicine, v. 367, n. 15, p. 1387-1396, Oct. 2012. Disponível em: $<$ http://www.nejm.org/doi/pdf/10.1056/NEJMoa1203039>. Acesso em: 17 ago. 2013.

SALINARDI, T. C.; BATRA, P.; ROBERTS, S. B.; URBAN, L. E.; ROBINSON, L. M.; PITTAS, A. G.; LICHTENSTEIN, A. H.; DECKERSBACH, T.; SALTZMAN, E.; DAS, S. K. Lifestyle intervention reduces body weight and improves cardiometabolic risk factors in worksites. The American Journal of Clinical Nutrition, v. 97, n. 4, p. 667-676, Apr. 2013. Disponível em: $<$ http://ajcn.nutrition.org/content/97/4/667.full.pdf + html $>$. Acesso em: 22 dez. 2014. 
THE WHOQOL GROUP. WHOQOL-bref: introduction, administration, scoring and generic version of assessment. Geneva: World Health Organization, 1996. Disponível em:

$<$ http://www.who.int/mental health/media/en/76.pdf $>$. Acesso em: 17 ago. 2013.

VENN, B. J.; MANN, J. I. Cereal grains, legumes and diabetes. European Journal of Clinical

Nutrition, v. 58, p. 1443-1461, May 2004. Crossef

WORLD HEALTH ORGANIZATION. Diet, nutrition and the prevention of chronic diseases: report of a joint WHO/FAO expert consultation. Geneva: World Health Organization, 2003.

Disponível em: <http://www.who.int/hpr/>. Acesso em: 22 set. 2013. 